\title{
A Method of Searching for Origins of Cosmic Rays correcting for Galactic Field Deflections and Charge Composition
}

\author{
Martin Erdmann, Gero Müller, Martin Urban*, Marcus Wirtz \\ Phys. Institute 3A, RWTH Aachen University, D-52056 Aachen, Germany \\ E-mail: erdmann@physik.rwth-aachen.de
}

\begin{abstract}
We present a new method of searching for origins of ultra-high energy cosmic rays directly from observed data. We include corrections for deflections in the galactic magnetic field according to the individual cosmic ray charges. The analysis procedure is iterative and consists of the following steps. Initially, we assign to each cosmic ray a charge hypothesis and apply corresponding corrections for the galactic field to obtain directions outside our galaxy. We then search for directions indicating an enhanced cosmic ray arrival probability using a clustering algorithm. The cluster directions form a set of source candidates. Hereafter, the initial charge assignments of the cosmic rays are disregarded, and a stacked source analysis is performed to evaluate the validity of the set of source candidates. The consistency of the observed cosmic rays with the expected arrival probability distributions on Earth is used in a likelihood ratio method on one hand for the evaluation of the set of source candidates, and on the other hand for assigning charges to each cosmic ray. The procedure can be repeated optimizing for the most likely set of sources. We present the method and its performance using a simulated astrophysical scenario.
\end{abstract}

35th International Cosmic Ray Conference

10-20 July, 2017

Bexco, Busan, Korea

\footnotetext{
${ }^{*}$ Speaker.
} 


\section{Introduction}

Since the discovery of cosmic rays 100 years ago their origin remains a question of debate. Rich data collections document the incoming particles on Earth with their direction, energy, and sometimes also particle type. At the highest energies measured thus far the cosmic particles are most likely protons and ionized nuclei [1,2].

Departures from isotropic distributions have been reported, e.g., a so-called hot spot [3], and a dipole signal [4]. The challenge of revealing directions of cosmic ray point sources may be due to extragalactic and galactic magnetic fields, which deflect charged particles depending on their electric charge, and thus complicate the reconstruction of point sources directly from the particle arrival directions.

In recent years, substantial progress has been made in the description of the galactic magnetic field. Based on Faraday rotation and synchrotron emission measurements, parameterizations have been developed that enable detailed predictions for the deflection of cosmic particles as a function of their energy and charge $[5,6,7]$.

In this contribution we present a new analysis concept to search for candidate directions of cosmic ray sources outside the magnetic field of our galaxy. Therefore a key element of the analysis are corrections for deflections of cosmic rays in the galactic magnetic field. The analysis strategy consists of two steps. In the first step the cosmic rays are assigned hypothetical charges to enable searches for candidate source directions outside the galactic field. In the second step the directions of the candidate sources are evaluated using the likelihood technique of a point source search.

This contribution is structured as follows. First we give an overview of the analysis strategy. Then we explain a simulated astrophysical scenario to explore the analysis method. In the following sections we discuss each step in detail before we evaluate the analysis method in different aspects. Finally we present our conclusions.

\section{Strategy}

The analysis strategy to search for candidate sources of cosmic rays is separated in two major steps and visualized in Fig. 1.

Charge assignment and search for source directions. The initial step includes the projection of cosmic ray directions to outside the galaxy and finding common directions in terms of clusters (points $1 \rightarrow 4$ in the figure). In the first point (1) of the figure our procedure assigns hypothetical charges to each cosmic ray randomly according to previous composition measurements [1].

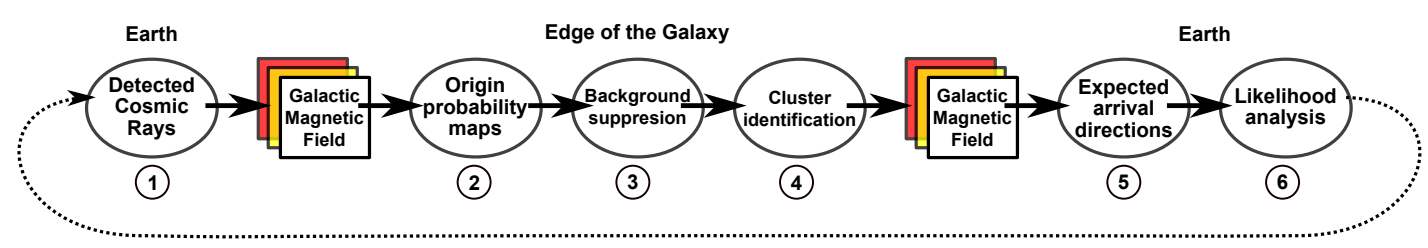

Figure 1: Strategy of the analysis. Between step 1 and 2 the data is projected to the edge of the galaxy and between step 4 and 5 from the edge of the galaxy to Earth. 
The cosmic ray directions are then corrected for deflections in the galactic magnetic field according to the charge assignment using a lensing technique (points $1 \rightarrow 2$ in the figure). The lenses will be explained below in section 3 . The result of the projection is a probability distribution of cosmic ray arrival directions outside our galaxy.

Instead of integer charge values we will use the continuous variable rigidity $R=E /(Z \cdot e)$ as the ratio of the cosmic ray energy $E$ and number $Z$ of elementary charges $e$.

With the cosmic rays also the coverage of the observatory is projected to the outside of our galaxy using the lenses with the same rigidities as for the cosmic rays. As the transparency of the galactic magnetic field is distributed inhomegeneously trivial spots of enhanced probability density will appear after the projection. In point (3) in the figure we keep only cosmic ray arrival probabilities that exceed the $90 \%$ quantile of the trivial arrival probability distribution.

Using the directions of all cosmic rays projected outside the galactic magnetic field a search for directions with high cosmic ray density is performed (point 4 in the figure). The search is performed with the DBSCAN algorithm which is one of the most common clustering algorithms for spatial data with noise [8]. The ensemble of directions with large densities then defines a point source scenario. It directly depends on the cosmic ray charge assignments as well as on the details of the galactic field description.

Evaluation of the point source scenario. In the second step the validity of this ensemble of source directions is evaluated like a standard point source analysis on a given catalog using the measured cosmic ray arrival directions (points $4 \rightarrow 6$ in the figure). In $4 \rightarrow 5$ the source candidate directions are projected onto the Earth for all relevant rigidity intervals. The probability distributions of the expected arrival are then used to evaluate the validity of the ensemble of source candidates in terms of a likelihood ratio measure (point 6).

We emphasize that in this evaluation step only the observed directions of the cosmic rays are needed. Neither the measured cosmic ray energies nor the hypothetical charge assignments are required for the evaluation of the point source candidates. Independently, the expected arrival distributions can be used to cross check the cosmic ray rigidity assignments (points $6 \rightarrow 1$ ).

Repetitive procedure. Note that our two-step procedure can also be repeated multiple times. For example, different sets of cosmic ray charge assignments can be used to check the stability of the source directions. Another possibility is to assign rigidities to the cosmic rays from the expected arrival distributions of the previous point source scenario. With this an iterative optimization procedure can be realized.

Usage of cosmic rays. In our procedure the cosmic ray directions are used on one side to search for source directions, and on the other side to evaluate the validity of an astrophysical scenario. If the observed cosmic rays originate from an isotropic distribution our procedure may generate source candidate directions as well. However, we will show below that if cosmic rays originate from distinct sources and our cosmic ray charge assignments and field corrections are correct to some extent the likelihood evaluation will be above trivial values. 


\section{Galactic magnetic field lenses}

In this contribution the regular magnetic field model from Jansson and Farrar [6] is used. Possible arrival directions on Earth of cosmic rays with different rigidities have been precomputed by backtracking antiprotons from Earth to the edge of the galaxy through the galactic magnetic field using the CRPropa v3 program [9]. The result of this simulation are lenses $l(R)$ describing the probability that a cosmic ray with rigidity $R$ entering the galaxy from direction $(l, b)$ can be observed at direction $\left(l^{\prime}, b^{\prime}\right)$ on Earth. Here $(l, b)$ are galactic longitude and latitude respectively. Correspondingly, the transposed lenses $l^{T}(R)$ can be used to project cosmic ray arrival directions on Earth to the outside of the galaxy. The lenses are stored using the HEALpix format [10] with nside $=64$ and lead to a substantial computational speedup compared to the direct usage of the galactic magnetic field model. In [11] we have shown that the rigidity should be greater than $6 \mathrm{EV}$ to remain in a regime of ballistic deflections. More details on galactic magnetic field lenses and their properties can be found in $[11,12]$.

\section{Simulated cosmic ray origin and observation}

For studying the analysis method we simulate an astrophysical scenario using active galactic nuclei with intense emission in the radio frequency regime as source directions [13]. We select three leading candidates according to their energy emission and distance which are FornaxA (NGC 1216), M87, and Centaurus A [14].

According to isotopic abundance in the solar system we use the top five nuclei hydrogen $(Z=1)$, helium $(Z=2)$, carbon $(Z=6)$, nitrogen $(Z=7)$ and oxygen $(Z=8)$ [15]. The Pierre Auger composition measurements [1] are used as a guide line to randomly assign a charge to each simulated cosmic ray. We use the relative probabilities $p(Z=1)=0.2, p(Z=2)=0.5$, and $p=0.1$ for each of the charges $Z=6,7,8$. The cosmic ray energy distribution follows a power law $\sim E^{-2.7}$. A lower energy cut is set at $E_{\min }=50 \mathrm{EeV}$ to ensure that the propagation through the galactic magnetic field is restricted to $R>6 \mathrm{EV}$ (see section 3). A smearing of $\delta=3^{\circ}$ around each source according to a Fisher distribution with concentration parameter $\kappa=1 / \delta^{2}$ is used to account for the extragalactic magnetic field and a random galactic magnetic field component.

In order to study the effect of background contributions we use a fraction of $15 \%$ of cosmic rays from the above mentioned sources, and assign random directions to the remaining $85 \%$ of the cosmic rays.

In fig. 2 we show the energy distribution and composition of the arriving cosmic rays. The number of events is set to 500 which is of the order of the event numbers in this energy regime measured by the Pierre Auger Collaboration [16]. We also show the arrival distribution of all cosmic rays in fig. 3 where the rigidities of the signal cosmic rays are color coded, and the background cosmic rays are shown in grey.

\section{Initial cosmic ray hypothetical rigidity and projection to outside the galaxy}

Usually, mass information is obtained from the longitudinal development of an air shower in the atmosphere, see e.g. $[2,17,18]$. In practice this information is not available for most of the 

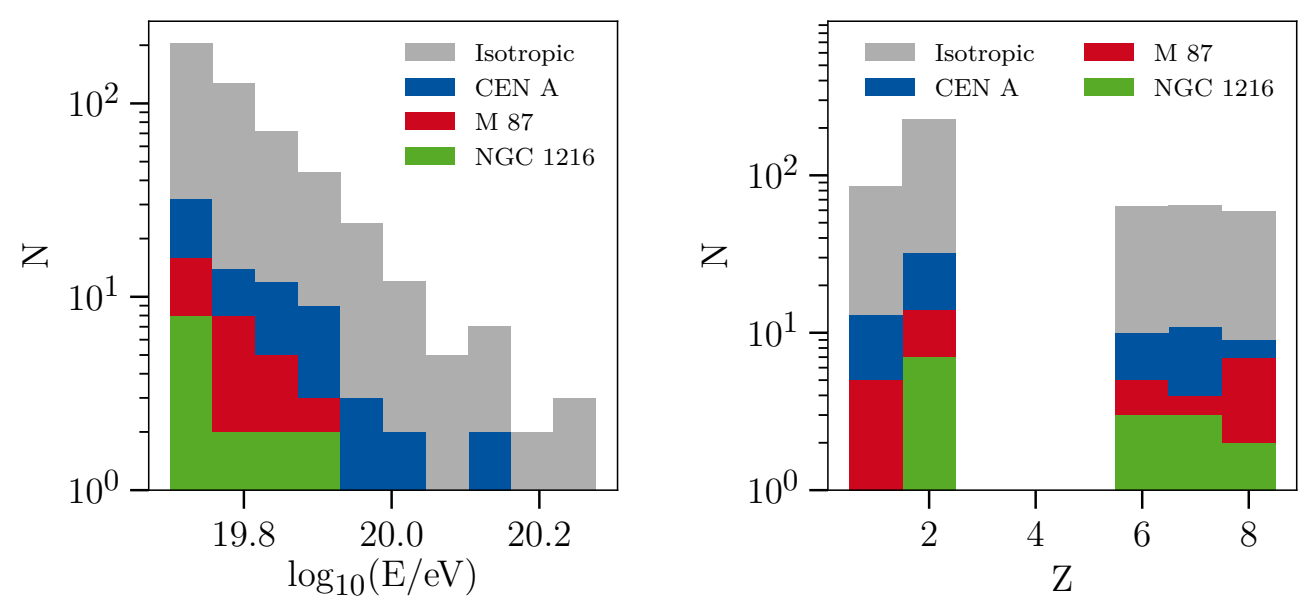

Figure 2: Energy distribution and composition of simulated cosmic rays.

highest energy events and thus a precise charge assignment is not feasible. Consequently, we assign each cosmic ray an equal probability for all charges between hydrogen and oxygen as an unbiased prior for this analysis. Using the cosmic ray energies we convert the charge probabilities to rigidity weights.

To perform the projection to outside the galaxy the cosmic rays are binned in rigidity with a bin width of $\log _{10} R=0.02$. Using the rigidity and the arrival directions of the cosmic rays for each rigidity interval a map of arrival probabilities is created. Due to the charge assignments cosmic rays contribute to different maps. Subsequently, the transposed lenses of the galactic magnetic field are used to transform these probability maps on Earth to outside the galaxy. As a final step the maps $x_{\text {data }}(R)$ at the edge of the galaxy are superimposed weighted with their total probability, see fig. 3. These maps are downgraded in the healpix pixeling scheme from nside $=64$ to nside $=16$ to reduce statistical fluctuations.

\section{Candidate directions of cosmic ray origins}

In this section we will use the above created projection map to the edge of the galaxy to identify source candidate directions. We evaluate the map for the simulated scenario by repeating the same analysis steps for 1000 isotropic sets. The obtained distributions in every extragalactic direction resulting from isotropic arrival on Earth are used to exclude regions of enhanced probability trivially arising from the inhomogeneous cosmic ray flux through the galactic magnetic field. A distribution of arrival probabilities summed over all rigidities is shown for an exemplary direction outside the galaxy in fig. 4. Only regions where the data value exceeds the $90 \%$ quantile of the isotropic expectation are used to search for high density regions.

High density regions are identified using the DBSCAN algorithm which can distinguish between clusters of different shapes and has good background rejection. For more details on the implementation see [8]. In figure 4 the centers $S_{\text {gal }}$ of the detected high density regions are shown by the green markers. The yellow stars denote the original source positions. 

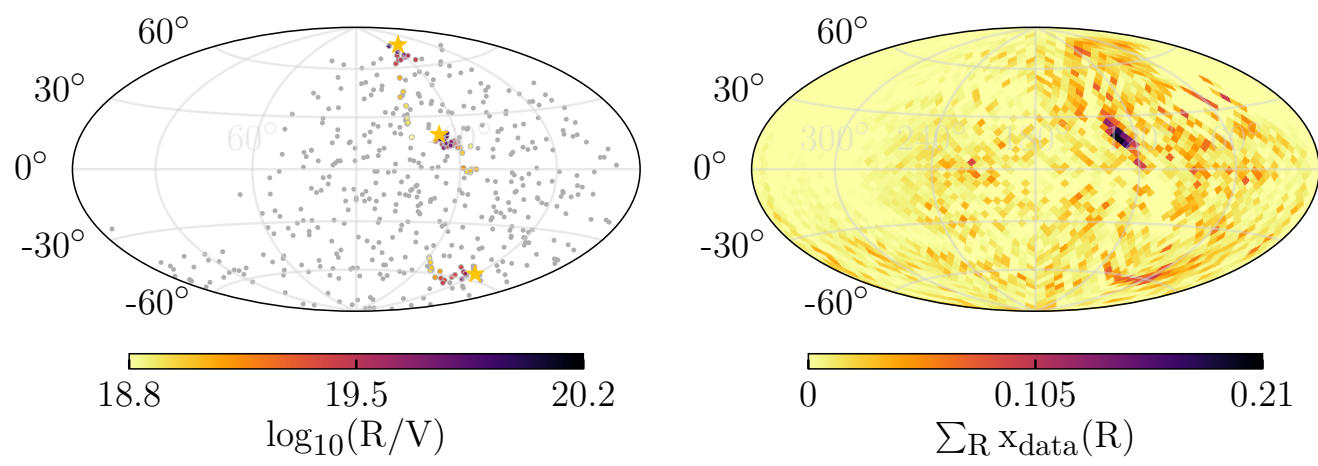

Figure 3: (left) Initial cosmic ray directions: isotropic background events in gray and signal events colorized by their rigidity. Yellow stars denote the directions of the AGN source (Fornax A, M87, Cen A). The geometric acceptance $A$ of the Pierre Auger Observatory is taken into account. (right) Sum of rigidity dependent projection of cosmic ray arrival directions on Earth to the edge of the galaxy: The color shows the summed probability in each pixel. Centaurus A is clearly visible, the other sources are less prominent. Both figures are in galactic coordinates.
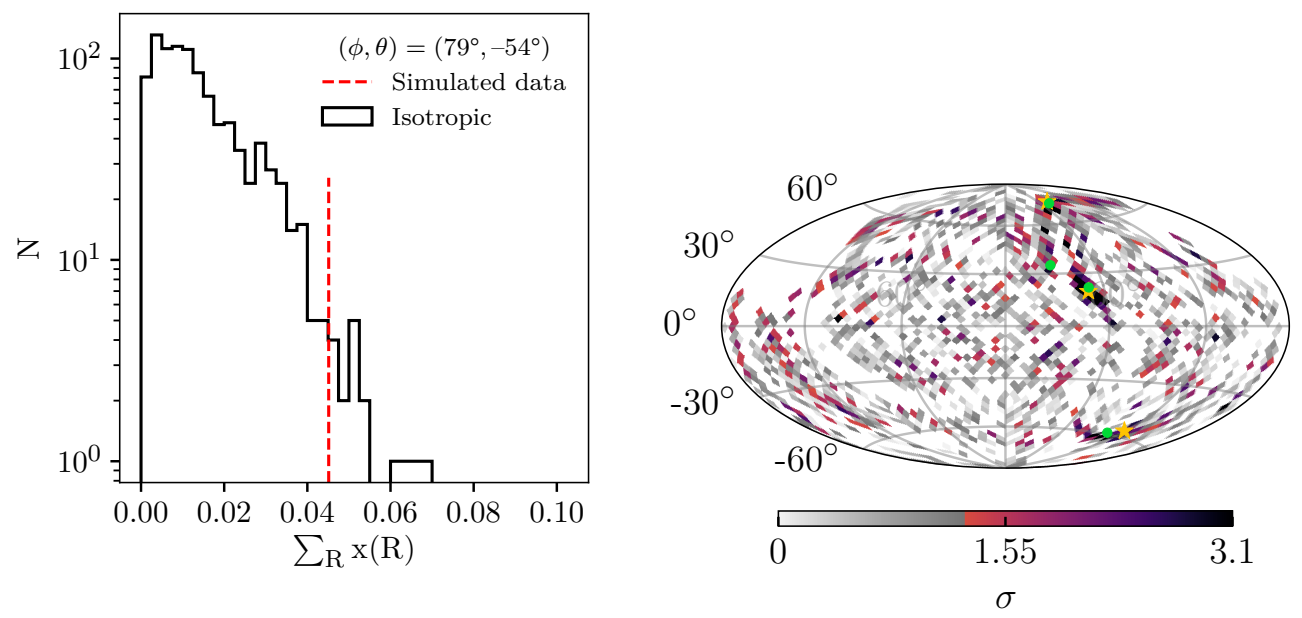

Figure 4: (left) Projected arrival probability distribution outside the galaxy for an exemplary direction. The simulated data value is shown by the vertical dashed line, and the distribution of isotropic arrival by the histogram. (right) Map containing deviations from the isotropic distributions. Directions exceeding the $90 \%$ quantile are colorized, the other directions are shown in gray.

\section{Expected arrival directions of cosmic rays}

The validity of the source candidates $S^{\text {gal }}$ obtained by the above search for clusters outside the galaxy is evaluated using the likelihood function,

$$
\mathscr{L}=\prod_{\mathrm{CRs}}\left[f \cdot S_{\delta}^{\text {Earth }}\left(l^{\prime}, b^{\prime}\right)+(1-f) \cdot B\left(l^{\prime}, b^{\prime}\right)\right]
$$

The underlying model consists of a a signal distribution $S_{\delta}^{\text {Earth }}$ on Earth and a background distribution $B$, both evaluated at the arrival directions $\left(l^{\prime}, b^{\prime}\right)$ of the cosmic rays. The variable $f$ denotes the 
anticipated signal fraction. The expected arrival distribution $S_{\delta}^{\text {Earth }}$ uses the lens $l(R)$ at different rigidities to calculate the projections of the source candidates $S^{\text {gal }}$ on Earth:

$$
S_{\delta}^{\mathrm{Earth}}=\int_{R_{\mathrm{CR}}} p_{\mathrm{CR}}(R) \cdot A \cdot l(R) \cdot S_{\delta}^{\mathrm{gal}} \mathrm{d} R
$$

The geometric acceptance $A$ of the Pierre Auger Observatory is taken into account [19]. We assume a Fisher distribution of concentration parameter $\kappa=1 / \delta^{2}$ around each candidate source to correct for extragalactic deflections of the cosmic rays and small scale random effects in the galactic magnetic field. The rigidity weight $p_{\mathrm{CR}}(R)$ ensures that each cosmic rays contributes with a total weight of one.

In fig. 5 the distribution of the test statistic $t=2 \log (\mathscr{L}(f, \delta) / \mathscr{L}(0,0))$ is shown. Both parameters, the signal fraction $f$ and the smearing $\delta$, have their maximum at the input values of the simulation. An isotropic scenario can significantly be excluded after a single pass through the analysis.
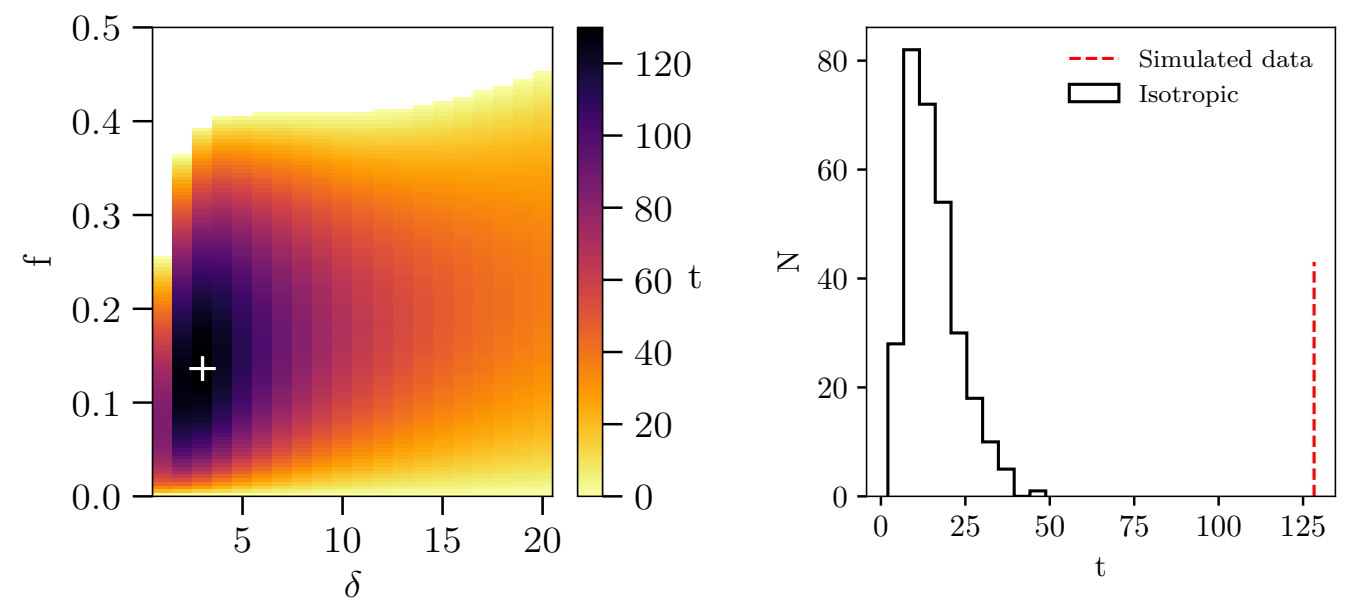

Figure 5: (left) Distribution of the test statistic $t$ which is shown by the color scale. The white cross marks the position of the highest value. (right) Test statistic of the simulated astrophysical scenario (vertical line) compared to isotropic scenarios after a single pass of the analysis procedure

The most probable charges of the signal cosmic rays were calculated by the rigidity $R$ maximizing the arrival distribution $S_{\delta}^{\text {Earth }}$ in eq. (7.2). For $60 \%$ of these cosmic rays the charges were correct or differed by $\Delta Z= \pm 1$.

\section{Conclusion}

We designed a method to identify source candidates from cosmic rays by charge dependent corrections for deflections in the galactic magnetic field. The method includes a search for extragalactic directions with enhanced arrival of cosmic rays. A likelihood ratio method is used to evaluate the validity of such source directions in comparison to isotropic scenarios. The projection of the identified source candidates on Earth can be used to constrain the charges of signal cosmic rays. 


\section{References}

[1] The Pierre Auger Collaboration, Depth of maximum of air-shower profiles at the Pierre Auger Observatory. I. Measurements at energies above $10^{17.8} \mathrm{eV}, 2014$, Phys. Rev. D, 90, no. 12, 122006

[2] The Pierre Auger Collaboration, Depth of maximum of air-shower profiles at the Pierre Auger Observatory. II. Composition implications, 2014, Phys. Rev. D 90, no. 12, 122005

[3] The Telescope Array Collaboration, Indications of Intermediate-Scale Anisotropy of Cosmic Rays with Energy Greater Than 57 EeV in the Northern Sky Measured with the Surface Detector of the Telescope Array Experiment, 2014, APJ., 790, L21

[4] The Pierre Auger Collaboration, Large Scale Distribution of Ultra High Energy Cosmic Rays Detected at the Pierre Auger Observatory with Zenith Angles up to 80, 2015, APJ., 802, no. 2, 111

[5] Pshirkov, M. S. et al., Deriving global structure of the Galactic Magnetic Field from Faraday Rotation Measures of extragalactic sources 2011, APJ., 738, 192

[6] Jansson, R., \& Farrar, G. R., A New Model of the Galactic Magnetic Field, 2012, APJ., 757, 14

[7] Beck, M. C. et al. New constraints on modelling the random magnetic field of the MW, 2016, JCAP, Issue 05 , article id. 056

[8] Ester, M. et al., A Density-Based Algorithm for Discovering Clusters in Large Spatial Databases with Noise, 1996, KDD-96, 226-231

[9] Batista, R. A. et al., CRPropa 3 - a Public Astrophysical Simulation Framework for Propagating Extraterrestrial Ultra-High Energy Particles, 2016, JCAP 1605, no.05, 038

[10] Gorski, K. et al., HEALPix - a Framework for High Resolution Discretization, and Fast Analysis of Data Distributed on the Sphere, 2005, APJ., 622, 759, 771

[11] Erdmann, M. et al., The nuclear window to the extragalactic universe, Astropart. Phys., 85, 2016, 54-64

[12] Bretz, H.-P. et al., PARSEC: A Parametrized Simulation Engine for Ultra-High Energy Cosmic Ray Protons, 2014, Astropart. Phys. 54, 110-117

[13] van Velzen, S., Falcke, H., All-sky catalog of local radio galaxies, The Intriguing Life of Massive Galaxies, Proceedings of the International Astronomical Union, IAU Symposium, 2012, Volume 295, pp. 271-271

[14] Müller, G., 2016, PhD thesis, RWTH Aachen University

[15] Cameron, A.G.W., Abundances of the elements in the solar system, 1973, Space Sci Rev 15: 121.

[16] The Pierre Auger Collaboration, Searches for Anisotropies in the Arrival Directions of the Highest Energy Cosmic Rays Detected by the Pierre Auger Observatory, 2015, APJ. 804, no.1, 15

[17] The Pierre Auger Collaboration, Evidence for a mixed mass composition at the 'ankle' in the cosmic-ray spectrum, 2016, Phys.Lett. B762 288-295

[18] The Pierre Auger Collaboration, Combined fit of spectrum and composition data as measured by the Pierre Auger Observatory 2017, JCAP04 038

[19] Sommers, P., Cosmic Ray Anisotropy Analysis with a Full-Sky Observatory, 2000, Astropart. Phys. 14,271 , 